\title{
Urgent surgical repair is pivotal in the management of major airway injury due to blunt trauma
}

\author{
Hysam Abdelmohty ${ }^{1}$, Mohamed A. Elanwar ${ }^{2}$ and Basem M. Abdelgawad ${ }^{3^{*}}$ (D)
}

\begin{abstract}
Background: Blunt traumatic airway injury is a life-threatening injury in which urgent management is pivotal and this would come through improving our clinical management and diagnostic tools. Our objective was to emphasize the importance of early referral and urgent surgical repair of major airway injury.

Results: Records of 42 patients with major airway injury out of 17,520 registered thoracic trauma cases were reviewed over the past 15 years. Twenty-eight cases documented to have major tracheobronchial injuries due to blunt trauma underwent surgical repair either urgent (21 cases) or late (7 cases). The age ranged from 8 to 43 years old with a mean age of $22.3 \pm 0.8$. The most common presenting symptom was shortness of breath in $22(78.6 \%)$ cases and the most frequent sign was subcutaneous emphysema which was seen in 22 (78.6\%) cases. The postoperative morbidity and mortality rates were significantly higher in the delayed repair group ( $p$ value $<0.001$ ).

Conclusion: The urgent surgical repair is the treatment of choice for major airway injury which is facilitated by early recognition and referral. It is pivotal to avoid respiratory and systemic complications and related mortality.
\end{abstract}

Keywords: Airway injury, Tracheobronchial tear, Surgical emphysema, Air leak

\section{Background}

Acute posttraumatic tracheobronchial injuries (TBI) are rare but potentially life-threatening events. It is responsible for nearly $30 \%$ of deaths at the scene [1]. The increasing incidence may be due to modernization, increased number of motor car accidents, work environment-related, or lately with the growing youth population competing in all sorts of sports. Rapid diagnosis and management are essential together with the proper assessment of associated injuries to set up the priorities in the ladder of management. Urgent surgical management of airway injuries prevents both early and late sequelae such as mediastinitis, airway stenosis, lobe collapse, and empyema.

The historical observation confirms that thoracic surgeons were aware of the airway injury as a swift killer, a

\footnotetext{
* Correspondence: bassem.abdelgawad@fmed.bu.edu.eg

${ }^{3}$ Cardiothoracic Surgery Department, Faculty of Medicine, Benha University,

15 Hasan Muwafi St., Hi Elrawdah, New Benha, Qalyobia 13518, Egypt

Full list of author information is available at the end of the article
}

century ago, and more. Despite that, the airway injuries still represent a difficult diagnostic and curative situation but fortunately, these injuries are rare. Some authors have reported that fatality is because of respiratory distress, difficulties in establishing an intact airway, and tension pneumothorax together with the high frequency of associated multiple precious organ injuries (head, spine, vascular, or parenchymal injuries) [2].

It is crucial to highlight the importance and the increasing incidence of major airway injury, and how to identify them among the great number of trauma patients. Furthermore, we emphasize the importance of early referral and urgent surgical repair of such injuries.

\section{Methods \\ Research design}

A retrospective comparative study reviewing records from the emergency hospital database over 15 years from January 2004 to January 2019. Data of 28 patients with

\section{Springer Open}

(๑) The Author(s). 2020 Open Access This article is licensed under a Creative Commons Attribution 4.0 International License, which permits use, sharing, adaptation, distribution and reproduction in any medium or format, as long as you give appropriate credit to the original author(s) and the source, provide a link to the Creative Commons licence, and indicate if changes were made. The images or other third party material in this article are included in the article's Creative Commons licence, unless indicated otherwise in a credit line to the material. If material is not included in the article's Creative Commons licence and your intended use is not permitted by statutory regulation or exceeds the permitted use, you will need to obtain permission directly from the copyright holder. To view a copy of this licence, visit http://creativecommons.org/licenses/by/4.0/. 
major airway injury due to blunt thoracic trauma who underwent surgical repair were studied. These patients were divided into two groups: the first group offered the urgent repair strategy and included 21 patients, and the second group was surgical repair was delayed and included seven patients.

\section{Inclusion and exclusion criteria}

The injuries involving the airway from below the cricoid cartilage until the secondary bronchial bifurcations were included. We have excluded injuries caused by penetrating trauma like stab wounds and also those caused by iatrogenic trauma such as rigid bronchoscope injuries. This decision was based on the higher trauma severity and multiple associated injuries accounting for the higher mortality in blunt trauma group compared to penetrating or iatrogenic groups thus making the results biased if the latter two groups were included in the study.

\section{Data management}

Details of demographic data, mode of injury, associated injuries, diagnostic modalities, management strategy, and outcomes were collected and retrospectively analyzed. We have collected the presenting symptoms and signs suggestive of airways injury. In stable cases, chest $\mathrm{x}$-ray (CXR), abdominal ultrasound (US), and computed tomography $(\mathrm{CT})$ of the chest; then, bronchoscopy of the airways were performed whenever possible. In hemodynamically unstable patients with shock or respiratory distress, resuscitation was performed according to the advanced trauma life support (ATLS) protocol; then, they were approached in the same manner as the stable patients or rushed to the operative theater for an exploratory thoracotomy.

\section{The protocol of management}

1. Indications for bronchoscopy. These were based on any clinical suspicion of significant airway injury like persistent pneumothorax with ineffective intercostal tube (ICT) drainage as witnessed by persistent high grade of air leak, presence of traumatic hemoptysis, progressive surgical or emphysema, and CT evidence of tracheal or bronchial discontinuity.

2. Resuscitation. Treating patients in shock, restoration of the reasonable airway, and alleviating pain

3. Monitoring. Continuous ECG monitoring, noninvasive blood pressure measurement, and obtaining peripheral oxygen saturation coupled with arterial baseline blood gasses

4. Anesthesia. Close co-operation between the anesthetist and the surgeon especially at the introduction of the endotracheal tube (ETT) that might need to be inserted with fiber-optic bronchoscopy. We have used a double-lumen ETT to facilitate healthy lung ventilation and to prevent blood spillover from the injured site. However, a single lumen tube was satisfactory in tracheal injuries.

5. Indications for surgery. The indications were evident significant $(>2 \mathrm{~cm})$ tear of the trachea or bronchi-detected bronchoscopically-as well as observing massive and persistent high degrees of air leakage from the thoracostomy tube and/or persistent acute respiratory distress.

6. Operative procedure

(1) Most of our patients had been explored via a classical posterolateral thoracotomy passing through the fourth intercostal space

(2) Careful inspection of the hemithorax was done with removing of collected blood, identifying the disturbed anatomy, and repairing any bleeding points.

(3) Identification of the tear edges and looking for any associated injuries

(4) Raw interrupted stitches were used to get the torn edges together after gentle debridement and reenforcement-when possible-by pleural or intercostals muscle flap.

(5) Testing for the integrity of the suture line and hemostasis. Then, two chest drains were inserted and thoracotomy was closed in layers.

7. Postoperative care. Our principle was to perform on-table or early extubation and encouraging selfventilation and self-exhaling of secretions together with proper physiotherapy. Early ambulation with strict physiotherapy was continued and a follow-up chest $\mathrm{x}$-ray was performed before discharge, and CT chest and/or bronchoscopy were done when the residual injury was suspected.

\section{Statistical analysis}

Statistical analysis data were statistically described in terms of mean, standard deviation $( \pm \mathrm{SD})$, frequencies (number of cases), and relative frequencies (percentages) when appropriate. A comparison of quantitative variables between the groups was done using the Mann-Whitney $U$ test, Chi-square $\left(\chi^{2}\right)$ test was performed for qualitative variables. A probability value ( $P$ value) less than 0.05 was considered statistically significant. All statistical calculations were done using computer programs Microsoft Excel version 7 and SPSS version 23 statistical program.

\section{Results}

Forty-two out of 17,520 registered thoracic trauma patients $(0.24 \%)$ reported having major airway injuries. 
Among those 42 patients, blunt trauma was the cause of injury in $31(73.8 \%)$ patients, while seven (16.6\%) injuries were due to iatrogenic causes, and only four $(9.52 \%)$ penetrating injuries.

Twenty-eight out of the 31 cases documented to have major TBI due to blunt trauma underwent surgical repair either early (21 cases) or late (seven cases). In our cohort, males were 22 and females six with a ratio of 3.7: 1 . The age ranged from 8 to 43 years old with a mean age of $22.3 \pm 0.8$.

The most common mode of injury in blunt trauma was the motor car accidents that were seen in 16 cases (57.1\%). While falling from height principally among working men was seen in four cases with a ratio of $14.3 \%$. Also falling of heavy objects on the chest either on-front or on-behind in four cases (14.3\%), raising concerns about their working environment. In our community, it is not uncommon to face animal attacks where a 17-year-old male received a kick by a calf to the front of the chest (3.6\%). Hit and run over a pedestrian by tricycle or motorbike was recorded in two cases $(7.1 \%)$ and one case (3.6\%) was registered sports injury.

Most of our cases arrived at the emergency room (ER) conscious but we did receive five cases (17.9\%) with a disturbed level of consciousness with the Glasgow coma scale between 9 and 13. Two of them (7.1\%) were admitted to our hospital already intubated by their referring hospitals, and three (10.7\%) were intubated in the ER. The most frequent presenting symptom and signs are shown in Table 1.

Chest radiographs were obtained in anteroposterior position, due to hemodynamic instability, disturbed level of consciousness, presence of masking surgical emphysema, and as a precaution for the possible cervical spine or orthopedic injuries, CT was done in almost all patients. The radiological findings as complete loss of the tracheal wall contour, displacement of the ETT, the air within the pericardium, mediastinal emphysema, pneumothorax, and lung collapse were detailed in Table 2 with examples in Figs. 1 and 2.

Tube thoracostomy was inserted in most cases at the primary hospital and a few of them at our ER. The unilateral tube was required in $18(64.3 \%)$ cases, a second apical tube was inserted in $4(14.3 \%)$ cases due to massive air leak and 6 (21.4\%) cases offered bilateral chest tubes. Bronchoscopy was diagnostic with the view of disrupted or lacerated tissues, continuous bleeding, and the presence of clots. Bronchoscopy was also useful in detecting the level of injury. We used primarily the rigid bronchoscopy except when its use becomes contraindicated in the presence of cervical spine injury. Bronchoscopy was not used in the six cases of upper tracheal injury where the neck tear was clear by the naked eye examination. Moreover, three cases with main stem bronchus injury were diagnosed by radiological findings (see Table 3).

Extra-thoracic injuries were common in severely traumatized patients. No significant injuries were collected and data are shown in Table 4. During surgical repair of the airways, two patients of the urgent group underwent evacuation of extradural hematoma and one patient underwent laparotomy for intra-abdominal collection.

We have used the previous data to suggest the criteria suspecting major TBI and indicating the use of bronchoscopy to confirm the diagnosis and to proceed for urgent surgical repair as detailed in Table 5.

We have used right thoracotomy in 16 cases, left thoracotomy in six cases, and cervical approach in six cases, where we have done 23 airway repairs, three lobectomies (10.7\%), and two pneumonectomies (7.1\%). None of our patients had the chance of emergency thoracotomy at the scene as this service is only available in tertiary centers. We did direct suturing to all cases after debridement of the tear edges. We had to do lobectomy in three cases $(10.7 \%)$ and pneumonectomy in two cases $(7.1 \%)$ due to severely damaged lung tissues.

Our patients were followed-up for periods ranged from 1 to 3 years with a mean follow-up period of $10.7 \pm$ 6.9 months with 7 (25\%) patients who had missed data during the follow-up. The early outcome during the follow-up period is shown in Table 6 .

\section{Discussion}

We had an incidence of 42 out of 17,520 registered thoracic trauma patients $(0.24 \%)$ reported having major airway injuries. The worldwide reported incidence in many works of literature is between 0.35 and $5.3 \%$. We can explain our incidence as cases that might be diagnosed as

Table 1 Clinical presentations

\begin{tabular}{|c|c|c|c|c|}
\hline Presenting symptoms & Total cases (28) & Urgent repair (21) & Delayed repair (7) & $P$ value \\
\hline Subcutaneous emphysema & $22(78.6 \%)$ & $17(81 \%)$ & $5(71.4 \%)$ & 0.34 \\
\hline Dyspnea & $22(78.6 \%)$ & $15(71.4 \%)$ & $7(100 \%)$ & 0.010 \\
\hline Hemoptysis & $9(32 \%)$ & $9(42.8 \%)$ & 0 & 0.023 \\
\hline Desaturation/cyanosis & $16(57.1 \%)$ & $14(66.6 \%)$ & $2(28.6 \%)$ & 0.027 \\
\hline Hoarseness of voice & $7(25 \%)$ & $6(28.6 \%)$ & $1(14.3 \%)$ & 0.045 \\
\hline Shock & $17(60.7 \%)$ & $15(71.4 \%)$ & $2(28.6 \%)$ & 0.039 \\
\hline
\end{tabular}


Table 2 Radiological findings and associated injuries

\begin{tabular}{lllll}
\hline Radiological findings: & Total cases (28) & Urgent repair (21) & Delayed repair (7) & P value \\
\hline Surgical emphysema & $27(96.4 \%)$ & $20(95.2 \%)$ & $7(100 \%)$ & $7(100 \%)$ \\
Pneumomediastinum & $23(82.1 \%)$ & $16(76.2 \%)$ & $4(57.1 \%)$ & 0.147 \\
Lung contusion & $22(78.6 \%)$ & $18(85.7 \%)$ & $4(57.1 \%)$ & 0.06 \\
Pneumothorax & $15(53.6 \%)$ & $11(52.4 \%)$ & $2(28.6 \%)$ & 0.019 \\
Hemopneumothorax & $9(32.1 \%)$ & $7(33.3 \%)$ & $2(28.6 \%)$ & 0.499 \\
Clavicle fracture & $9(32.1 \%)$ & $7(33.3 \%)$ & $1(14.3 \%)$ & 0.499 \\
Flail chest & $8(28.6 \%)$ & $7(33.3 \%)$ & $2(28.6 \%)$ & 0.025 \\
Fallen lung sign & $6(21.4 \%)$ & $4(19 \%)$ & & 0.419 \\
\hline
\end{tabular}

a major airway injury but dies before surgery is conducted. Besides, the cases that passed unregistered or undiagnosed by the trauma team were not obvious and required a high degree of suspicion.

Most studies report that the most common cause of TBI is blunt trauma. In the present study, TBI was caused by blunt trauma in $73.8 \%$ of trauma cases. Approximate figures were reported by many authors such as Richardson [3], Scaglione et al, [4], and Koletsis et al. [5] who had found, respectively, that $76.7 \%, 67 \%$, and $66.7 \%$ of injuries were due to blunt trauma. However, few authors reported their cases to be caused mainly by penetrating trauma such as Fatimi et al. [6] and Saad et al. [7]. We think that the accuracy of these figures was biased by the small sample size in most of the studies.

In our study, the age ranged from 8 to 43 years old with a mean age of $22.3 \pm 0.8$ and 11 patients (39.3\%) were under 18 years old. Similar results were reported by Balci et al. [8] and Saad et al. [7] as they had a mean age

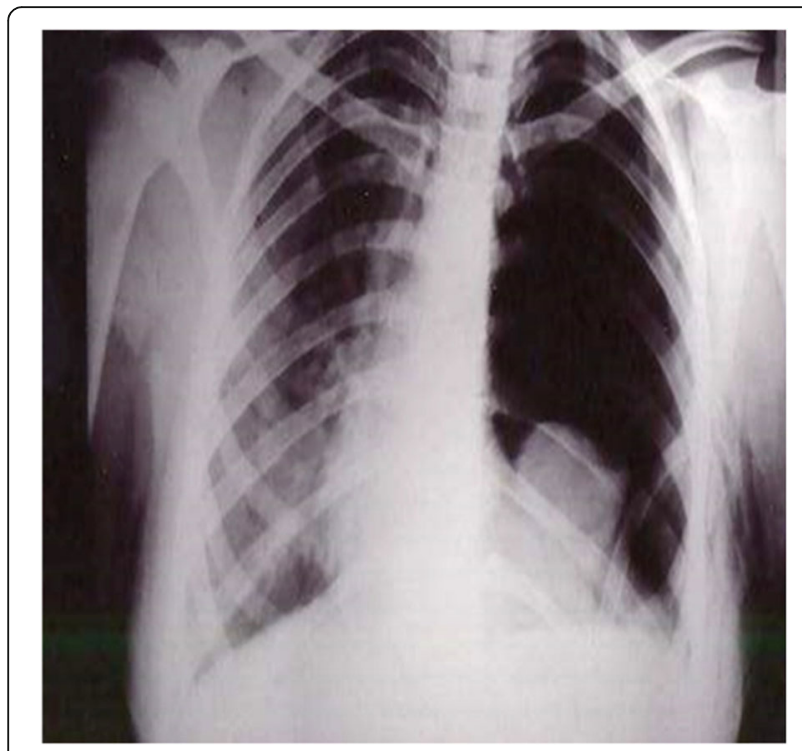

Fig. 1 Fallen lung sign of kumpe of 22.3 years (range $4-53$ ) and 26 years (range 17-38), respectively.

Several mechanisms causing tracheal injury have been suggested: tracheal compression between the sternum and the vertebral column or abrupt increase in intraluminal pressure due to forced expiration against a closed glottis [9]. Direct trauma to the cervical trachea also causes tracheal injury [10]. Tracheobronchial injuries involve mostly the main bronchus within $2 \mathrm{~cm}$ close to the carina; the right bronchus is more commonly affected [11]. Chest compression squeezes the right main bronchus that is seated in front of the vertebral bodies and increases the risk of tear during blunt incidents, while the left main bronchus gets protection by the arch riding above it. Also, the longer air column with the left bronchus buffers the increasing intraluminal pressure and reduces the tear incidence [10].

In our cohort, the injuries were more common at the upper airways especially the cervical part of the trachea and this finding was consistent with almost all previous reports. Some authors like Farzanegan et al. who studied 35 patients with major airway injury and included 16 (45\%) of them with a laryngeal injury [12]. We have excluded such patients as they are out of our scope of care being managed by the ENT surgeons.

Injuries ranged from contusion, a small laceration, to complete avulsion of the airways. Once the injury violates the integrity of the mediastinal pleura, it will produce persistent air leak and desaturation, but if it remains intact, it might go unnoticed as both ends are connected by the peribronchial tissues but later on stenosis endures and patients come with dyspnea at rest [9].

In our population, surgical emphysema was the most frequent presentation followed by dyspnea. Many authors reported the same findings even with more distress in the form of tachypnea and stridor $[8,13,12,5]$. The incidence of hemodynamic instability and shock was $60.7 \%$ which is higher than those documented in many studies as we included only cases of blunt trauma that commonly involved other systems. 


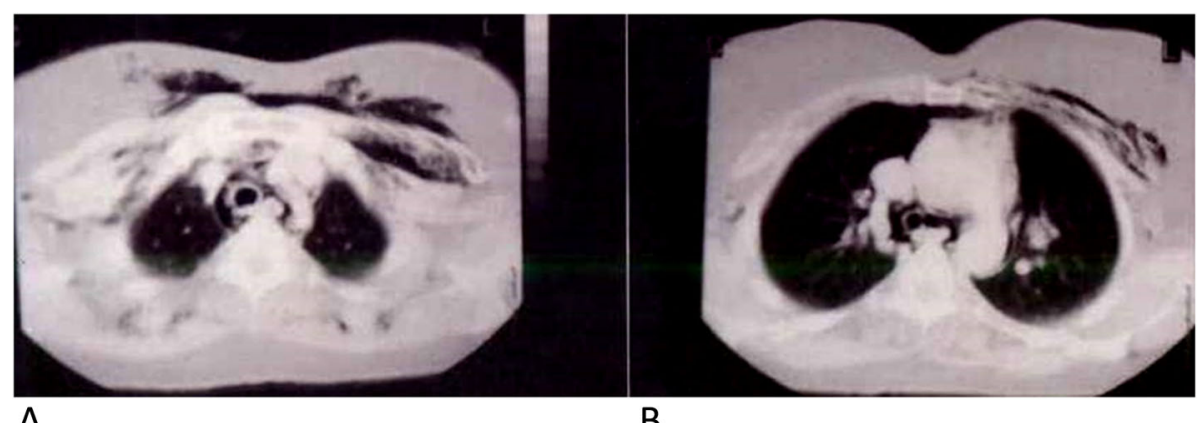

A

B

Fig. 2 a, b Air encircling the trachea till carina and around vessels, pneumomediastinum, and surgical emphysema

In our study, pneumothorax was present in $24(85.7 \%)$ of cases and lung contusion was reported in 22 (78.6\%) of them. Few patients displayed no signs or symptoms until one or 2 days post-injury [10[. Serious associated other injuries included multiple ribs fracture, flail chest, and head or abdominal injuries; they occur in more than $50 \%$ of patients as similarly reported by others [14].

The chest CT is able to detect the site of blunt tracheobronchial injuries in more than $90 \%$ of cases. However, bronchoscopic examination remains the standard tool for diagnosing TBI not only to detect but also to define the site and the extent of any tear or laceration [15]. Bronchoscopic findings may be in the form of single transverse (between the rings) or longitudinal along the membranous segment or maybe complex with rupture in more than one site or form [16].

Immediate surgical repair was chosen in most cases to avoid airway and pulmonary complications. In a few selected cases, surgical intervention was postponed if the patient did not have major injury suspicious criteria or did not have a large tracheobronchial tear by bronchoscopic examination. The latest ATLS guidelines recommended the immediate operative intervention in such injuries except for more stable patients; operative treatment of TBI may be delayed until the acute inflammation and edema resolve [2]. Although conservative treatment may be an option in selected patients, it is crucial to bear in mind that it may be a one-way choice, as the success of subsequent surgical treatment in case of failure may be limited by the progression of sepsis, reduced vascularization of the margins of the airway laceration, and the development of adhesions [17].

The following is a decision-making flow chart for managing chest trauma with keeping major TBI in mind:

A cervical tracheal tear can be approached by collar cervical incision while right posterolateral thoracotomy provides good access to the intrathoracic trachea, right main bronchus, and proximal part of the left main bronchus [17]. Some authors reported a high rate of pneumonectomy, $23 \%$, and referred that to the high rate of gun-shot injuries [3].

All cases were managed by direct repair after trimming of tear edges without much dissection to keep reasonable blood supply to the suture lines. We have used absorbable $3 / 0$ interrupted sutures with ties outside and in few cases; we covered the suture line with mediastinal pleura. Using of absorbable sutures in repairing of the tracheal tear is best with less chance for the formation of granulation tissue than the non-absorbable suture. Also, it is liable to cause abscess, infection, or dehiscence. Interrupted sutures with knots outside are commonly performed than the continuous technique. The suture line can be supported by Teflon or a pleural flap [18].

Postoperative use of bronchoscopic examination, nasotracheal suction, or forced coughing is important, and the patients should be reassessed by bronchoscope 3 months after repair [18, 19]. Tracheal stenosis is

Table 3 The site of the tracheobronchial tear

\begin{tabular}{lllll}
\hline Site of the tear & Total cases (28) & Urgent repair (21) & Delayed repair (7) & $P$ value \\
\hline Upper half of trachea & $6(21.4 \%)$ & $6(28.6 \%)$ & 0 & $3(42.8 \%)$ \\
Main stem bronchus & $8(28.6 \%)$ & $5(17.9 \%)$ & $4(19.1 \%)$ & $3(42.8 \%)$ \\
Lower trachea extending to Rt main bronchus & $7(25 \%)$ & $3(14.3 \%)$ & $1(14.3 \%)$ & 0.071 \\
Lower trachea extending to Lt main bronchus & $4(14.3 \%)$ & $3(14.3 \%)$ & 0 & 0.218 \\
Complex injury & $3(10.7 \%)$ & & 0.982 \\
\hline
\end{tabular}


Table 4 Extra-thoracic injuries

\begin{tabular}{|c|c|c|c|c|}
\hline Extra-thoracic injuries & $\begin{array}{l}\text { Total cases } \\
(28)\end{array}$ & $\begin{array}{l}\text { Urgent repair } \\
\text { (21) }\end{array}$ & $\begin{array}{l}\text { Delayed repair } \\
\text { (7) }\end{array}$ & $\begin{array}{l}P \\
\text { value }\end{array}$ \\
\hline Severe head trauma* & $8(28.6 \%)$ & $6(28.6 \%)$ & $2(28.6 \%)$ & 0.98 \\
\hline $\begin{array}{l}\text { Abdominal organs injury with Intra-abdominal collection or capsular } \\
\text { hematoma }\end{array}$ & $8(28.6 \%)$ & $5(17.9 \%)$ & $3(42.8 \%)$ & 0.071 \\
\hline Long bones fracture & $14(50 \%)$ & $10(47.6 \%)$ & $4(57.1 \%)$ & 0.037 \\
\hline Pelvic fracture & $3(10.7 \%)$ & $3(14.3 \%)$ & 0 & 0.019 \\
\hline Spinal cord injury & $2(7.1 \%)$ & $2(9.5 \%)$ & 0 & 0.03 \\
\hline
\end{tabular}

*Severe head trauma: associated with intracranial hemorrhage, fracture base, or post-concussion symptoms with disturbed consciousness

diagnosed if the diameter decreased to $50 \%$ and clinically it does dyspnea only on exertion but if it reaches below one quarter, stridor and dyspnea will occur at rest especially if little secretions or inflammatory swelling was seated inside [17].

In our patients, the postoperative morbidity and mortality rates were significantly higher in the delayed repair group ( $p$ value $<0.001$ ). These rates were affected by the longer time of lung collapse, lung and surgical site infection, and presence of retained hemothorax. We had three cases of tracheal stenosis; one of them responded to multiple bronchoscopic dilatations, and the other two needed surgery for disabling dyspnea. The two empyema cases in the urgent repair group managed by tube thoracostomy drainage and medical treatment and showed a complete cure. The only case of empyema in the delayed repair group died after developing septic shock. One case with massive air leakage with significant lung collapse underwent early thoracoscopic repair. The other two cases required no further intervention rather than physiotherapy and observation. They improved and

Table 5 Criteria raising suspicion of major tracheobronchial injury

A. Criteria in the early presentation or during conservative treatment

History Severe chest trauma especially to the upper part, neck trauma

Severe dyspnea, voice change

Symptoms

Signs Progressive surgical emphysema, cyanosis, shock

X-ray Mediastinal emphysema, fallen lung

CT Endotracheal cuff herniation, tracheal wall discontinuity,

findings bronchus discontinuity, displacement of the ETT, overdistension of the endotracheal cuff

After ICT Massive air leakage with the failure of lung re-expansion

B. Criteria in late presentation

History of severe chest trauma with continuing dyspnea, recurrent chest infection, lung consolidation or collapse discharged after 2 weeks. Local granulation appeared over the anastomosis in two patients and was successfully removed with the bronchoscope.

There were non-comparable reasons for mortality in both groups. In the urgent repair group, the mortality of three cases was attributed to the involvement of other organ damage; two cases died because of brain injury, one case because of increased abdominal bleeding and shock after thoracic surgery and the fourth one died after adult respiratory distress syndrome (ARDS). In the delayed group, one patient developed complicated acute renal failure and the other two patients died after septic shock; one of them due to pneumonia after surgical airway repair and the other patient due to prolonged empyema.

The mortality varied according to the anatomical location of the airway injury, studies show a higher rate of fatality if the thoracic portion of the trachea was injured compared to the cervical part and higher mortality if injury with lower bronchus is injured [4]. Because it is difficult to ventilate/oxygenate, even after tracheal intubation and mechanical ventilation, and even get worsened [18]. Thus, those who survive the accident were those with upper injuries while those who die at the scene were those with lower injuries [19].

\section{Conclusion}

Bronchoscopic examination remains the standard tool for diagnosing major tracheobronchial injuries. We recommend urgent surgical repair for the treatment of major airway injury to avoid infections and other complications. The conservative management must be reserved for smaller tears in stable patients and should be reverted to surgery upon the worsening of clinical or radiological findings.

\section{Study limitation}

Our limitations included the retrospective nature of the study depending on the available amount of data contained in the computer database. Missing data like 
Table 6 Outcome of surgical repair

\begin{tabular}{|c|c|c|c|c|}
\hline & Total cases (28) & Urgent repair (21) & Delayed repair (7) & $P$ value \\
\hline Suture line dehiscence/prolonged air leakage* & $3(10.7 \%)$ & $1(4.8 \%)$ & $2(28.6 \%)$ & $<0.001$ \\
\hline Empyema & $3(10.7 \%)$ & $2(9.5 \%)$ & $1(14.3 \%)$ & \\
\hline Airway stenosis & $3(10.7 \%)$ & $1(4.8 \%)$ & $2(28.6 \%)$ & \\
\hline Endobroncheal granulation tissue & $2(7.1 \%)$ & $1(4.8 \%)$ & $1(14.3 \%)$ & \\
\hline Mortality & $7(25 \%)$ & $4(19.1 \%)$ & $3(42.8 \%)$ & \\
\hline
\end{tabular}

*Prolonged air leakage: more than seven days of air leakage

airway management at the scene and the follow-up data were not complete with short-term follow-up period. The co-relation of death and the airway injury was determined subjectively as usually there are no detailed nor clear statements in our patients' files on the exact scenario of death.

\section{Abbreviations}

TBI: Tracheobronchial injuries; CXR: Chest x-ray; US: Ultrasound; $C T$ : Computed tomography; ATLS: Advanced trauma life support; ICT: Intercostal tube; ETT: Endotracheal tube; ER: Emergency room; ARDS: Adult respiratory distress syndrome

\section{Acknowledgements}

Not applicable.

\section{Authors' contributions}

-HEA, MAE, and BMA: (1) Substantial contributions to conception and design, acquisition of data, or analysis and interpretation of data; (2) drafting the article or revising it critically for important intellectual content; and (3) final approval of the version to be published. HEA and BMA: Data collection and analysis, methodology development, and manuscript writing.

\section{Funding}

No external source of funding.

\section{Availability of data and materials}

Data are available on request.

\section{Ethics approval and consent to participate}

Ethics approval of the study protocol was approved by the Ethical Committee of the Faculty of Medicine at Cairo University Hospital. Committee's reference number is not applicable. The need for informed consent was waived due to the retrospective nature of the study.

\section{Consent for publication}

Not applicable.

\section{Competing interests}

No conflict of interest and nothing to disclose.

\section{Author details}

${ }^{1}$ Cardiothoracic Surgery Department, Faculty of Medicine, Mansoura University, Mansoura, Egypt. ${ }^{2}$ Cardiothoracic Surgery Department, Faculty of Medicine, Cairo University, Cairo, Egypt. ${ }^{3}$ Cardiothoracic Surgery Department, Faculty of Medicine, Benha University, 15 Hasan Muwafi St., Hi Elrawdah, New Benha, Qalyobia 13518, Egypt.
2. Henry S, Brasel K, Stewart RM (2018) Advanced trauma life support, student course manual Tenth edition. American College of Surgeons. 65

3. Richardson JD (2004) Outcome of tracheobronchial injuries: a long term perspective. J Trauma. 56:30-36

4. Scaglione M, Romano S, Pinto A, Sparano A, Scialpi M, Rotondo A (2006) Acute tracheobronchial injuries: impact of imaging on diagnosis and management implications. Eur J Radiol. 59:336-343

5. Koletsis E, Prokakis C, Baltayiannis N, Apostolakis E, Chatzimichalis A Dougenis D (2012) Surgical decision making on the basis of clinical evidences and the injury's anatomical setting. Injury. 43:1437-1441

6. Fatimi SH, Hanif HM, Ahmed A, Shamsi G, Muzaffar M (2011) Outcomes of surgical management of tracheobronchial injuries - a case series from a developing country. Chinese J Traumatol. 14(3):161-164

7. Saad R Jr, Gonçalves R, Perlingeiro JA DNVG, Rivaben JH, Botter $M$ et al (2017) Tracheobronchial injuries in chest trauma: a 17-year experience. Rev. Col. Bras.Cir. 44(2):194-201

8. Balci AE, Eren N, Eren S, Ulku R (2002) Surgical treatment of post-traumatic tracheobronchial injuries: 14-year experience. European Journal of Cardiothoracic Surgery. 22:984-989

9. Milner A (2008) The airway in face, head, and neck injury. SAJAA. 14:52-57

10. Nelson LA (2007) Airway trauma. Int Anesthesiol Clin. 45:99-118

11. Kummer C, Netto FS, Rizoli S, Yee D (2007) A review of traumatic airway injuries: potential implications for airway assessment and management. Injury. 38:27-33

12. Farzanegan $R$, Alijanipour $P$, Akbarshahi $H$, Abbasidezfouli A, Pejhan $S$, Daneshvar A et al (2011) Major airways trauma, management and long term results. Ann Thorac Cardiovasc Surg. 17:544-551

13. Rossbach MM, Johnson SB, Gomez MA, Sako EY, Miller OL, Calhoon JH (1998) Management of major tracheobronchial injuries: a 28 year experience. Ann Thorac Surg. 65:182-186

14. Savas $R$, Alper $H$ (2008) Fallen lung sign: radiographic findings. Diagn Interv Radiol. 14:120-121

15. Scaglione M, Romano S, Pinto A et al (2006) Acute tracheobronchial injuries: impact of imaging on diagnosis and management implications. Eur J Radiol. 59:336-343

16. Iwasaki M, Kaga K, Ogawa J, Inoue H, Shohtsu A (1994) Bronchoscopy findings and early treatment of patients with blunt tracheo-bronchial trauma. J Cardiovasc Surg. 35:269-271

17. Carretta A, Melloni G, Bandiera A, Negri G, Voci C, Zannini P (2011) Conservative and surgical treatment of acute posttraumatic tracheobronchial injuries. World J Surg. 35:2568-2574

18. Glazer ES, Meyerson SL (2008) Delayed presentation and treatment of tracheobronchial injuries due to blunt trauma. J Surg Educ. 65:302-308

19. Hurford WE, Peralta R (2003) Management of tracheal trauma. Can J Anesth. 50(suppl):R1-R6

\section{Publisher's Note}

Springer Nature remains neutral with regard to jurisdictional claims in published maps and institutional affiliations.

Received: 29 June 2020 Accepted: 10 September 2020

Published online: 18 September 2020

\section{References}

1. Schneider T, Volz K, Dienemann H, Hoffmann H (2009) Incidence and treatment modalities of tracheobronchial injuries in Germany. Interact Cardiovasc Thorac Surg. 8:571-576 